\title{
THE MORPHOLOGIES OF SNRS AND THEIR ISM AND CSM
}

\author{
ZHENRU WANG \\ Dept. of Astronomy, Nanjing Univ., Nanjing 210093, PR China
}

Recently, many unusual morphologies of supernova remnants (SNRs) have been discovered: semicircular shell; center-brightened but without center point source; two-lobed; bipolar; irregular, etc. To understand these varieties of morphologies, we have made theoretical models. One of the main reasons of their unusualness is the multiphase structure of the interstellar medium (ISM) and circumstellar medium (CSM) where they evolved, and the CSM is connected with their progenitor.

The morphologies of SNRs were classified into three types-shell, plerion and composite types (Weiler 1985). It made progress in understanding SNRs.

We are interested in the irregular SNRs discovered recently that deviated from the above standard shapes. The most obvious example is the famous semicircular shell of CTB 109. It has been explained by our model of a SN explosion occured near the interface between a molecular cloud and diffuse ISM (Wang et al. 1992a). Another interesting example is IC443. Its morphology in soft $\mathrm{X}$-xays is very different from that in the radio and optical (Petre et al. 1988). Its hard X-rays up to $20 \mathrm{kev}$ has been observed by Ginga (Wang et al. 1992b). Considering IC443 expanding into a multiphase CSM and ISM, the very hard X-ray component is reasonably contributed from its western part. The age of IC443 was deduced to be in the range of 1000-1400 yrs, consistent with the explosion of the Tang Dynasty SN AD837 (Wang et al. 1992b, 1993). The joint ROSAT-Ginga observation further supported our above conclusion (Asaoka \& Aschenbach 1994) because of the fact that the new SNR G189.6+3.3 discovered recently by ROSAT is not at the same distance as IC443.

Einstein, ROSAT and ASCA have discovered many center brightened SNRs without central point sources, e.g., G292.0+1.8, Kes 79, Kes 27, G18.9-1.1, HB9, G299.2-2.9, G272.2-3.2, RXJ 1713.7-3946 and W49B. W49B is center-brightened, but the other $8 \mathrm{SNRs}$ are center-ring-brightened. It is 
obvious that they did not fit easily into the simple three-type classification scheme. White and Long (1991) considered SN evolving in to a homogeneous intercloud medium with a uniform cloud distribution to explain its center -brightened shape. We (Chen, Liu \& Wang 1995) have considered SNR expanding into an intercloud stellar wind bubble. The resulting thermal $\mathrm{X}$-ray emission has a center-ring-brightened morphology. We conclude that the remnants of $\mathrm{SN} \mathrm{Ib} / \mathrm{c}$ can be center-ring-brightened even if no central point source is visible.

In the radio band, many $S N R s$ have unusual morphologies. For example, G292.0+1.8 and G357.7-0.1 have center-brightened shapes with steep spectra but no central point sources, G76.9+1.0, Kes 79, G18.9-1.1, G93.7-0.3, and G65.7+1.2 have double-lobed or bipolar morphologies and so on.

The importance of the CSM to the evolution and non-thermal radio emission for the remnants of SN Ib/c and SN II is also obvious. We (Zhang, Wang \& Chen 1996) have considered the evolution of SNRs in the circumstellar wind cavities. The freely expanding and shocked wind regions are the zones in this kind of SNRs that are most likely to be observed in the radio band. The magnetic structure of these two regions is likely to have toroidal configuration (Chevalier 1992). We expect that this orderly toroidal magnetic structure should affect the distribution of radio emission. Synchrotron radiation should be diminished in those parts of the remnant where the direction of the magnetic field route is parallel to the line of sight. As a result, the apparent morphologies will vary with aspect angles as follows (Zhang, Wang \& Chen, 1996): center-brightened when observed in the equatorial plane; shell type when observed near the polar direction and two-lobed or bipolar when observed in the medial directions.

\section{References}

Asaoka, I. and Aschenbach, B. 1994, A\&A, 284, 573

Chen, Y., Liu, N. and Wang, Z. R. 1995, ApJ, 446, 755

Chevalier, R. A. 1992, ApJ, 397, L39

Petre, R., Szymkoviak, E., Seward, F. D. and Willingale, R. 1988, ApJ, 335, 215

Wang, Z. R., Qu,Q. Y., Luo, D., McCray, R. and Mac Low, M. M., 1992a, ApJ, 388, 127

Wang, Z. R., Asaoka, I., Hayakawa, S. and Koyama, K. 1992b, PASJ, 44, 303

Wang, Z. R. 1993, in UV and X-ray Spectroscopy of Laboratory and Astrophysical Plasma, eds. Silver and Kahn (Cambridge Univ. Press), p. 407

Weiler, K. W. 1985, in The Crab Nebula and Related Supernova Remnants, eds. Kafatos and Henry, (Cambridge Univ. Press), p. 265

White, R. L. and Long, K. S. 1991, ApJ, 373, 543

Zhang, Q. C., Wang, Z. R. and Chen, Y, 1996, ApJ, 466, 808 